Relations industrielles

Industrial Relations

\title{
Wages in Practice and Theory, by Robert Ozanne, the University of Wisconsin Press, Madison, 1968, 181 pages.
}

\section{L.-René Parenteau}

Volume 24, numéro 2, 1969

URI : https://id.erudit.org/iderudit/028033ar

DOI : https://doi.org/10.7202/028033ar

Aller au sommaire du numéro

Éditeur(s)

Département des relations industrielles de l'Université Laval

ISSN

0034-379X (imprimé)

1703-8138 (numérique)

Découvrir la revue

Citer ce compte rendu

Parenteau, L.-R. (1969). Compte rendu de [Wages in Practice and Theory, by Robert Ozanne, the University of Wisconsin Press, Madison, 1968, 181 pages.] Relations industrielles / Industrial Relations, 24(2), 453-454.

https://doi.org/10.7202/028033ar

Tous droits réservés @ C Département des relations industrielles de l'Universite Laval, 1969
Ce document est protégé par la loi sur le droit d'auteur. L’utilisation des services d’Érudit (y compris la reproduction) est assujettie à sa politique d'utilisation que vous pouvez consulter en ligne.

https://apropos.erudit.org/fr/usagers/politique-dutilisation/ 
transposées comme telles dans de nouvelles conventions, ce dont les auteurs demeurent conscients. Dans leur esprit, il s'agit plutôt de modèles à partir desquels il est possible de construire de nouvelles clauses adaptées aux situations concrètes.

Toutefois comme le domaine de la convention collective en est un qui évolue très vite, les clauses, tant dans leur contenue, que dans leur formulation, connaissent des changements constants. C'est pourquoi les auteurs ont prévu que l'ouvrage (présenté dans un cahier à feuilles mobiles) serait remis à jour périodiquement. C'est d'ailleurs à cette seule condition qu'il pourra continuer de rendre les services pour lesquels il a été voulu.

\section{Jean BERNIER}

Labor Law, vol. I, by Joseph Alton Jenkins, The W. H. Anderson Company, Cincinnati, 1960, 528 pages.

Le droit du travail aux Etats-Unis est si complexe, son évolution est tellement liée à l'histoire économique et politique de ce pays qu'il est parfois difficile de s'y retrouver ou même de le considérer comme une branche isolée des autres parties du droit. Il ne faut pas oublier non plus les problèmes particuliers que soulèvent l'élaboration et l'application d'un droit du travail dans un état fédéral tel que les Etats-Unis.

C'est dans ce contexte que l'auteur entend faire une étude, la plus complète possible, de la législation du travail dans ce pays en la restituant dans son cadre historique et constitutionnel.

Le premier tome aborde trois grands ordres de problèmes seulement. D'abord les aspects constitutionnels du droit du travail aux Etats-Unis de même que les juridictions des divers tribunaux. La deuxième partie est consacrée à une étude du «National Labor Relations Board $\gg$. Enfin, le dernier chapitre traite des causes de « représentation 》 ou accréditation syndicale devant le «N.L. R.B. », notamment la procédure, la détermination de l'unité de négociation appropriée, le vote, etc.

Les explications sont en général brèves et concises. Par ailleurs, il est intéressant de noter que l'ouvrage, en plus de faire état d'une jurisprudence abondante, rapporte le texte intégral d'un grand nombre de documents qui en constituent un utile complément. C'est ainsi qu'on $\mathrm{y}$ trouve les principales lois du travail, des documents et des formules officielles de N.L.P.B.

L'ensemble constitue un instrument de travail précieux pour le praticien comme pour le professeur ou le chercheur.

Avant de porter un jugement global sur l'ouvrage, il sera intéressant de prendre connaissance des trois tomes à venir qui porteront sur les «pratiques déloyales » tant de la part de l'employeur que de la part du syndicat et sur certaines autres questions relatives aux injonctions, aux conflits d'urgence nationale, à la poursuite en dommage et le reste.

Jean BERNIER

Wages in Practice and theory, by Robert Ozanne, the University of Wisconsin Press, Madison, 1968, 181 pages.

Le professeur Ozanne nous propose une étude propre à l'économique du travail et aux relations industrielles. Par une étude de cas, il nous amène à reconsidérer, par la pratique, des hypothèses fondamentales des théories du salaire et des théories du comportement. Nous devons peut-être à Robert Ozanne une des premières grandes études de cas dans le domaine des relations industrielles. L'expérience est d'une valeur extrêmement importante, car elle annonce possibles de multiples études du genre après environ 100 ans de pratique des relations industrielles; elle présente aux relations industrielles une autre méthode de recherche, méthode qu'ont déjà utilisée les sciences plus anciennes.

Grâce à la collaboration de la famille McCormick, le professeur Ozanne a pu se constituer, à partir des feuilles de paie et de la correspondance de la compagnie (McCormick and International Harvester), une histoire des relations industrielles dans cette compagnie. Les résultats étonnent: d'abord, nous avons là reconstitué l'histoire statistique des variations de salaires entre 1860 et 1960 , histoire située dans un contexte économique national. L'auteur a donc pu étudier les rapports entre les grandes crises 
économiques, la syndicalisation, le développement des marchés et les fluctuations de salaires dans la compagnie. Ensuite, à l'intérieur même de la compagnie, les rapports statistiques indiquaient à l'auteur les rapports entre les augmentations de salaires et les accroissements de la productivité, les rapports entre la syndicalisation des ouvriers et les augmentations de salaires, rapports qu'il a su vous présenter dans les termes des théories classiques et modernes des salaires. Ainsi, dans sa conclusion, le professeur Ozanne met les salaires en rapport avec quatre grandes variables d'une théorie des salaires, soit la syndicalisation, la demande de travail sur le marché, la rigidité des échelles salariales, la productivité et le marché du produit.

Malheureusement, la valeur de ce travail est remise en question à cause de la spécificité du sujet à l'étude. En effet, si nous ne pouvons généraliser les conclusions, à quoi nous servirons les études empiriques? Le professeur Ozanne pose lui-même la question et, bien que brièvement, il $\mathrm{y}$ répond par la localisation de la compagnie ( $\ll$ urban center $)$ et par l'influence d'un syndicat (《 city-wide ») dont le champ dépassait les seuls cadres de l'entreprise. Pour maintenant, ces justifications doivent nous suffire.

Nous ne doutons pas cependant que plusieurs études de ce genre sont maintenant rendues possibles grâce aux développements de l'histoire et que leurs conclusions, comme celles de Robert Ozanne, apportent les assises empiriques et les généralisations scientifiques que nécessite la pratique des relations industrielles.

\section{René PARENTEAU}

Manpower planning for High Talent Personnel, par Eric W. Vetter, Bureau of Industrial Relations The University of Michigan, 1967, 222 pages.

La détermination des besoins en main d'oeuvre est une activité qui occupe une place importante dans la gestion rationnelle des ressources humaines. Elle fait l'objet d'une section ou d'un chapitre dans les volumes nouveaux ou dans les dernières éditions d'anciens volumes en administration du personnel au sein de l'entreprise privée.
On peut se rendre compte du nombre d'articles publiés sur ce sujet depuis le début de la présente décade en jetant un coup d'oeil sur la longue liste qui constitue l'essai bibliographique. Le volume apparaît comme un véritable effort de synthèse et de réflexion. Le but est de fournir un guide aux dirigeants et aux spécialistes de la gestion du personnel qui sont peu familiers avec la conception, la conduite et l'évaluation d'un effort de planification ou détermination des besoins de cadres supérieurs, intermédiaires et subalternes. Les premiers chapitres portent sur la nature et les phases $\mathrm{du}$ processus. On y retrouve aussi une description détaillée de la procédure à suivre et une application de cette procédure dans l'industrie de l'aéronautique. Le quatrième et le cinquième chapitres portent sur des points plus précis tels que l'établissement d'un inventaire de main d'oeuvre hautement spécialisée et la façon de mesurer la productivité. Avec le chapitre VI, on apprend à établir des prévisions en main d'oeuvre en partant du calcul du rendement anticipé. Le chapitre suivant décrit la démarche qui permet de relier les prévisions aux objectifs de rentabilité de la firme pour la période envisagée par le plan. Le préposé à la planification doit être en mesure d'évaluer les résultats des efforts déployés; il doit aussi être capable de définir sa place et son rôle au sein de l'organisation qui l'emploie. Les deux derniers chapitres traitent brièvement de ces questions.

Le dirigeant et le spécialiste du personnel doivent rendre compte que toute activité de recrutement, sélection, placement, formation, promotion devient beaucoup plus facile et efficace lorsqu'elle est précédée d'un effort de planification. C'est pourquoi, il aurait intérêt à lire rapidement ce volume qui ne présente aucune difficulté technique.

\section{Laurent BELANGER}

La mobilité des travailleurs urbains, par L. Hunter et G. Reid, OCDE, Paris, 1968, 239 pages.

A une époque où les progrès de la technologie et l'évolution des structures sont aussi rapides, la mobilité de la main-d'oeuvre devient indispensable. Si la mobilité actuelle de la main-d'oeuvre n'est pas suffisante, une intervention des 